\title{
Molecular Profiling of Microbial Communities from Contaminated Sources: Use of Subtractive Cloning Methods and rDNA Spacer Sequences
}

Frank T. Robb

Center of Marine Biotechnology

410-234-8870

701 E. Pratt Street

Baltimore MD 21202

410-234-8896 (FAX)

robb@umbi.umd.edu

\section{Research Objective}

The major objective of the research was to provide appropriate sequences and to assemble a DNA arrays of oligonucleotides to be used for rapid profiling of microbial populations, from polluted areas and from areas of other interest. The sequences to be assigned to the DNA array are chosen from cloned genomic DNA from groundwater at DOE sites containing organic solvents. The sites, Hanford Nuclear Plant and Lawrence Livermore Site 300 (LLNL), have well characterized pollutant histories, which have been provided by our collaborators.

Methods

We are developing microarrays using the 16s ribosomal DNA intergenic spacer regions (ISRs) to profile community composition, for rapid characterization of microbial communities in contaminated ecosystems, and for detection of bacteria carrying pollutant-metabolizing genes. This DNA sequence and our routine methods have been described in our previous reports. Glass slide arrays were made and tested as follows. Hypervariable stretches of the ISRs, in the 16s-to-tRNA-ILE, inter-tRNA (itRNA), and tRNA-ALA- to-23s regions, were synthesized and hybridized against synthetic templates and PCR products corresponding to the opposite strand in collaboration with Orchid (nee Molecular Tool, Inc). Capture probes for silanized glass slide hybridization experiments were modified with a 5' sulfhydryl group for attachment to the slides. Detection probes were modified with 3' fluorescein tags; synthetic templates were unmodified. PCR products were produced using one primer with a 5' fluorescein and four 5' phosphorothioate bonds, followed by 0.5-1 hour at $37 \cdot \mathrm{C}$ in the presence of $1 \mathrm{unit} / \mu \mathrm{L} \mathrm{T} 7$ gene 6 exonuclease to make them single stranded. This exonuclease digests double stranded DNA, working from the 5' end of each strand; the strands with the phosphorothioate linkage were therefore resistant to this enzyme.

Arrays on glass slides were made by spotting capture probes and fluorescent marker in 500 $\mathrm{mM} \mathrm{pH} 9$ bicarbonate buffer (either $0.1 \mu \mathrm{L}$ by hand or $30 \mathrm{~nL}$ by Hamilton 220 automated pipetting robot), allowing attachment overnight, washing 3 times in TNTw, overlaying with 1 pmol synthetic template or $0-5 \mathrm{pmol}$ PCR product that had been heated in the presence of detection probe 10 minutes at $95 \cdot \mathrm{C}$ and then cooled to $37 \cdot \mathrm{C}$. Following a $0.5-3$ hour hybridization, the slides are washed 3 times in TNTw and scanned on a Molecular Dynamics Fluorimager using a $530 \mathrm{~nm}$ filter. Results

In order to distinguish progress of acetate and nitrate bioremediation in the Hanford area, it appears that 4 sequences (replicated) are all that are required. The data reveal considerable strain variation between sample sites. The distribution of sequences found is striking: From 38 to $45 \%$ of the unique sequences are found only once in each level. The presence of three of the common clones, 1.02, 2.04, and 4.01, is correlated with the process of remediation: 1.02 appears at the end, 2.04 early (predominantly), and 4.01 early or in the middle of remediation. These three clones are from pseudomonads, as are 20 of all the bins (according to blastn). Other bins blast best against 
Thiobacillus, Agrobacterium, Bartonella, Xanthomonas, and Xylophilus. We assume little PCR bias based on the generality of the primers used and on the use of at least three pairs of primers per sampling site. We are synthesizing diagnostic oligos for as many of the Hanford bins as possible in order to compare the ISRs found in the LLNL sites, and to establish the microarray method. The optimal time (3 hours) of hybridization and the detection limit ( $10 \mathrm{fmol})$ using PCR products of plasmid DNA .

Monitor wells within the LLNL Building 834 Area having varying amounts of VOC contamination are being sampled for groundwater microbiological assessment. Five monitor wells have been selected for this study based on their VOC concentrations. Two of the wells selected (W834-D3, and W-834-D14) have high VOC concentrations, predominantly TCE, in excess of 100,000 $\mu \mathrm{g} / \mathrm{L}$. A third well (W-834-B3) has lower levels of VOCs, around 10,000 $\mu \mathrm{g} / \mathrm{L}$, which is almost entirely composed of 1,2-DCE. The two remaining wells selected (W-834-S6, and W-834-T5) have not historically contained any VOC compounds (clean wells, control populations). All five wells selected are installed within the same hydrologic unit.

ISRs from 2 LLNL wells have been sequenced, again revealing a striking difference in the bacterial flora between moderate and high VOC levels, as shown in Fig. 1. The more contaminated groundwater appears more diverse at this stage of sequencing. There appear to be several identical signature ISRs at both the Hanford and LLNL sites.

\section{Bead-based method for hybridization detection}

A new multiplexed, bead-based method which utilizes nucleic acid hybridizations on the surface of microscopic polystyrene spheres to identify specific sequences in heterogeneous mixtures of DNA sequences was developed. The method consists of three elements: beads (5.6-microm diameter) with oligomer capture probes attached to the surface, three fluorophores for multiplexed detection, and flow cytometry instrumentation. Two fluorophores are impregnated within each bead in varying amounts to create different bead types, each associated with a unique probe. The third fluorophore is a reporter. Following capture of fluorescent cDNA sequences from environmental samples, the beads are analyzed by flow cytometric techniques which yield a signal intensity for each capture probe proportional to the amount of target sequences in the analyte. In this study, a direct hybrid capture assay was developed and evaluated with regard to sequence discrimination and quantitation of abundances. The target sequences (628 to $728 \mathrm{bp}$ in length) were obtained from the $16 \mathrm{~S} / 23 \mathrm{~S}$ intergenic spacer region of microorganisms collected from polluted groundwater at the nuclear waste site in Hanford, Wash. A fluorescence standard consisting of beads with a known number of fluorescent DNA molecules on the surface was developed, and the resolution, sensitivity, and lower detection limit for measuring abundances were determined. The results were compared with those of a DNA microarray using the same sequences. The bead method exhibited far superior sequence discrimination and possesses features which facilitate accurate quantitation

\section{Student Training}

For the past year Karen Schindler, an undergraduate student from Loyola College working with our collaborator Dr. Mary Lowe, has been sequencing the LLNL samples, both as work-study in the summers and as a research project during the academic year. 


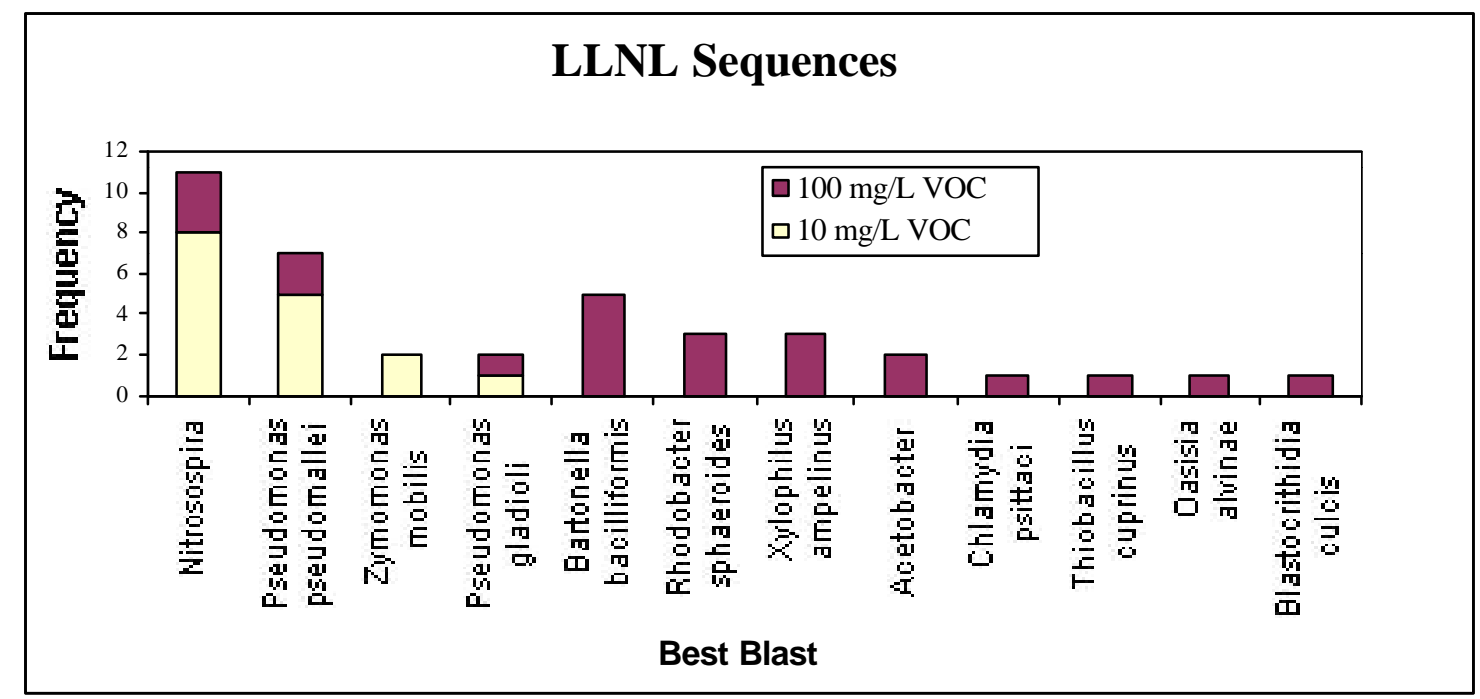

Figure 1. Distribution of ISR sequences in LLNL samples representing high and moderate VOC levels.

\section{Conclusions}

Our data so far from Hanford suggests that diversity does vary greatly with bioremediation and that there are many bacterial ISR sequences can indicate the effects of bioremediation. We will shortly test the environmental DNA from LLNL (as a PCR amplified sample) in an inter-site hybridization experiment against the sequences from the Hanford sites to determine overlap in microbial community composition between the sites. We will also hybridize against sequences described in the NCBI and Deep Subsurface databases, to probe for sequences without the intermediate cloning step. We have recently made arrangements to acquire more samples from the Hanford site (Evan Dressel, pers. comm.). The samples are from the same geologic strata and include clean well samples. We will test PCR products against the arrays to find which bacterial strains are common to both Hanford areas, and, after subtractive hybridization, concentrate on novel ISRs.

We chose the 3' end of the $16 \mathrm{~S}$ for one (universal) primer and the probe; the other (diagnostic) primer will come from the ISR within 150 bases from the first primer. By assembling a distribution of strains from LLNL as from Hanford we have been able to identify several cosmopolitan groundwater strains that are probably solvent resistant. Because bacterial communities in groundwater have been difficult to study until the advent of molecular techniques, we have contributed to an understanding of how diverse these environments are, and we have devised new molecular techniques to describe them. 


\section{Publications:}

Spiro A, Lowe M, Brown D (2000) A bead-based method for multiplexed identification and quantitation of DNA sequences using flow cytometry. Appl Environ Microbiol 66(10):4258-65.

Lowe, M, Halden,R Schindler,,K Smith, C, Emrich, S Frank Robb, F.T. and E. L. Madsen. (2001) Geochemical and Molecular Microbial Characterization of a Trichloroethene-Contaminated Subsurface Site Undergoing In Situ Reductive Dechlorination. Appl. Environ, Microbiol., in preparation.

Brown, D.C., Holley-Shanks, R., Maeder, D.L., Lowe, M.L., Rogers, Y-H., Brockman, F., and F. T. Robb (2001) Rapid Turnover of Microbial Communities in Carbon Tetrachloride-contaminated Groundwater during Bioremediation: Use of rDNA Intergenic Spacer Regions for Strain Identification. Appl. Env. Microbiol. In preparation

\section{Acknowledgements and Contact Information}

This project is supported by DOE Environmental Management grant

Telephone 410-234-8870,8871; email robb@umbi.umd.edu,, maeder@umbi.umd.edu. Web Site: http://comb5-156.umbi.umd.edu/bags.html (local ISR database) 